\title{
AN AGENT-BASED MODELLING FOR RIDE SHARING OPTIMIZATION USING A* ALGORITHM AND CLUSTERING APPROACH
}

\author{
M. Naseri Gorgoon ${ }^{1}$, M. Davoodi ${ }^{2, *}$, M. Davoodi ${ }^{3}$, H. Motieyan ${ }^{4}$ \\ ${ }^{1}$ M.Sc. in GIS at Faculty of Civil Engineering, Islamic Azad University Ramsar Branch, Iran - naseri.mng@ gmail.com \\ ${ }^{2}$ M.Sc. in GIS at Faculty of Geodesy and Geomatics, K.N.Toosi University of Technology, Iran - mojtaba.davoodi@ut.ac.ir \\ ${ }^{3}$ B.Sc. in Electrical Engineering at Electrical and Computer School, Electrical Engineering Department, University of Ghiaseddin \\ Jamshid Kashani, Qazvin, Iran - e.mohsen.davoodi@gmail.com \\ ${ }^{4}$ Department of Geo-Spatial Information System, Faculty of Geomatic and Geodesy, University of K.N.Toosi of Technology, \\ Tehran, Iran - H.motieyan@ sina.kntu.ac.ir
}

\section{Commission VI, WG VI/4}

KEY WORDS: Ride Sharing, Urban Traffic, Agent-based Modelling, Clustering, NetLogo

\begin{abstract}
:
Today, city management is one of the great challenges facing the world. The growth of population, industries, and services is in urgent need of transportation on a large scale. Meanwhile, transportation has great importance in urban management. Therefore, it is necessary to solve the traffic problem with scientific methods and reduce the traffic load of cities. An interesting way to reduce urban travels is using 2,3, or 4 people from one car that it is known as "Ride Sharing". In this research, the NetLogo software is used to simulate travel sharing scenarios. The three considered parameters are the number of passengers, the acceptable travel sharing radius, and the acceptable waiting time. The proposed algorithm uses a clustering method to find the best candidates to share a ride. Several scenarios were performed to evaluate numerical results. The number of passengers was 100 , and 500 , the radius of the trip was 1,000 and 2,000 meters, and the waiting time was 10 and 20 minutes. So, 8 experiments were carried out. The least amount of travel sharing was observed in the first scenario (100 passengers, $1000 \mathrm{~m}$ travel sharing radius and 10 minutes waiting time), in which $2 \%$ of single trips dropped out. The most sharing trips were in the final scenario (500 passengers, 2000 meters radius and 20 minutes waiting time), which saw a decrease of $36.4 \%$ of single trips. So, it can be said that sharing a trip can reduce traffic in cities and consequently reduce urban costs and either air pollution or noise pollution.
\end{abstract}

\section{INTORIDUCTION}

The development of cities and the increase of urban life in the present century, along with the growth of industry and the economic power of countries, has created and emerged new problems. One of these problems is increasing traffic in cities. So, a lot of costs, time, and fuel are wasted, and all these costs are imposed on the whole society. Therefore, it is necessary to manage existing resources and use scientific methods to overcome these problems. In this regard, one of the existing solutions is the sharing of travel between travelers. The carsharing includes a service provider that allows drivers and travelers to share their travels with similar travelers' plans, thus they can share the costs (Charles and Kline, 2006). Car sharing systems combine the benefits of speed, comfort, and flexibility (like personal vehicles) with the low cost of public transportation systems such as buses and subways. Increasing the number of passengers in a car for a trip and efficient use of car capacity will have a significant impact on reducing environmental pollution, fuel consumption, and cost savings.

\section{LITERATURE REVIEW}

Lin et al developed a model that concentrates on the routing optimization of ride-sharing taxis, in which minimization of charges and maximization of customer satisfaction are regarded as the objective. Besides, travel mileage, waiting time, and extra riding time due to ride-sharing are applied to quantify them respectively. Consequently, the computational analysis indicates that the proposed model can preserve $19 \%$ mileage as well as $66 \%$ taxis available (Lin et al., 2012).

Santos and Xavier studied an optimization problem that resembles conditions including either dynamic ride-sharing or taxi-sharing. Not only the information of passengers such as their origin, destination, the earliest departure, the latest arrival time, and the maximum cost they can pay for the ride is reported by an application, but also either car proprietors or taxi operators determine their location, destination, the leaving time, the maximum acceptable standing time, and a price per kilometer. To solve this dynamic obstacle, the day is split into several periods. For each period, an instance of a static problem is created, and solved by a greedy randomized adaptive search procedure (GRASP). The results depict that passengers can save up to $30 \%$ on shared travels compared to private trips (Santos and Xavier, 2015).

Nourinejad and Roorda evaluated the performance of Agentbased modeling $(\mathrm{ABM})$ for dynamic ridesharing problem. In this research, a vicinity approach supports potential matching choice sets for either drivers or passengers. The agent-based model gives close to optimal results within short computation times. The results demonstrate higher user cost savings and vehicle kilometers traveled (VKT) savings when allowing multi-passenger rides. Besides, short term revenue is maximized by a commission rate of roughly $50 \%$ (Nourinejad and Roorda, 2016).

Stiglic et al examined improving urban mobility by combining ride-sharing and public transportation that contribute fast, reliable, and affordable transfer to and from transportation stations in rural areas. Although the computational operations 
note that connecting ride-sharing and public transit can significantly enhance urban mobility, driver willingness to serve more than one rider is crucial for success (Stiglic et al., 2018). Simonetto et al introduced a novel, computationally efficient, a dynamic algorithm to simulate real-time city-scale ridesharing via linear assignment problems. The algorithm is based on a linear assignment problem and a federated optimization architecture. Besides, it allows high computational efficiency and quality of service. Real-time ridesharing is shown to offer clear benefits even with partial adoption. In multi-company scenarios, negative effects could arise in terms of the higher number of vehicles (Simonetto et al., 2019).

\section{METHODS AND MATERIALS}

In this research, it was expected to receive passenger's travel information from the Snap or TAP30 company (real information), and the model would be applied to the information of those trips to determine how much travel sharing can be beneficial in case of cost and time-saving. Unfortunately, this information was not received, and inevitably the simulated information was used. In other words, a series of origindestination and random request times were produced as passenger information.

All passenger's travel request information includes the origin, destination and time of travel request were received. Then, an AI-based algorithm attempts to find common routes between passengers who have close origins and destinations, then with a slight change in the route of two passengers, they create a common route and take the second passenger, and It will optimally take both passengers. At the end of the scenario, the number of travels and the length of trips are compared with the scenario that every passenger travels individually, and the effectiveness of this method is examined.

Since agent-based modeling has been used in this study, the agents are travelers who understand their position and other passengers (Zhang and Levinson, 2004); indeed, they transform their information with each other and find out where are the other agents (travelers).

The agent-based modeling simulations can be divided into two categories: 1) Single-agent or 2) Multi agents. For instance, a taxi can be considered as two separate agents including taxi driver and passenger. To simplify the simulation, we assume the taxi driver and the passenger as one agent. It means, each passenger has a vehicle who wants to travel through the network, but if it is possible based on some conditions, it will be combined with another passenger, and they use one vehicle. The architecture of agent-based modeling simulations is divided into four main categories: 1) Logic-based, 2) Reactive, 3) Belief Desire Intention, and 4) Layered (Bellifemine et al., 2007). Therefore, the architecture of this simulation is simple, singleagent, and logic based. There are three rules for agents to share their trip: 1) Closeness of their origins, 2) Closeness of their destinations, and 3) Closeness of their travel request time.

Then, they decide in the sharing of the trip after applying the three conditions of the closeness of origin, destination, and travel time. Applying three conditions for sharing is done by the clustering method. Besides, the passengers who are the agents choose to share their ride or travel individually.

After determining which trips are performed shared or individually, the routing operation is performed, and the system outputs including the number of travels and the total length of trips, are obtained, and the system is evaluated.

\subsection{Route finding using $A^{*}$ algorithm}

In this paper, $\boldsymbol{A}^{*}$ algorithm is used for the routing process. This algorithm uses a heuristic method to determine the optimal path between two points. Not only $\boldsymbol{A}^{*}$ algorithm selects the next node based on the least cost from the initial vertex like Dijkstra algorithm (Dijkstra, 1959), but it also uses an estimation about the distance to the destination point (Yao et al., 2010). One of the most common estimations corresponding to the total length between origin and destination is Euclidean distance. The cost function for $\boldsymbol{v}$ vertex in a graph with $\boldsymbol{s}$, first vertex, and $\boldsymbol{t}$, the final vertex is (Choset et al., 2005):

$$
\begin{aligned}
& f(v)=d(v)+h(v) \\
& f(v)=d(v)+\sqrt{(x(v)-x(t))^{2}+(y(v)-y(t))^{2}}
\end{aligned}
$$

Where $f(v)$ is the cost function between $s$ to $v,(x(v), y(v))$ is the coordinates of $v,(x(t), y(t))$ is the coordinates of final point of algorithm, $d(v)$ is the length of the route between $s$ to $v$ and $h(v)$ is the Euclidean distance between $v$ to $s$.

The calculation of the optimal path is the same as the Dijkstra algorithm. Searching for the shortest path in this algorithm, in contrast to the Dijkstra algorithm that extends radially from the starting point, is based on the end point, and therefore the processing time is much less than the Dijkstra algorithm.

The steps in this algorithm is depicted in Figure 1 (Zidane and Ibrahim, 2017).

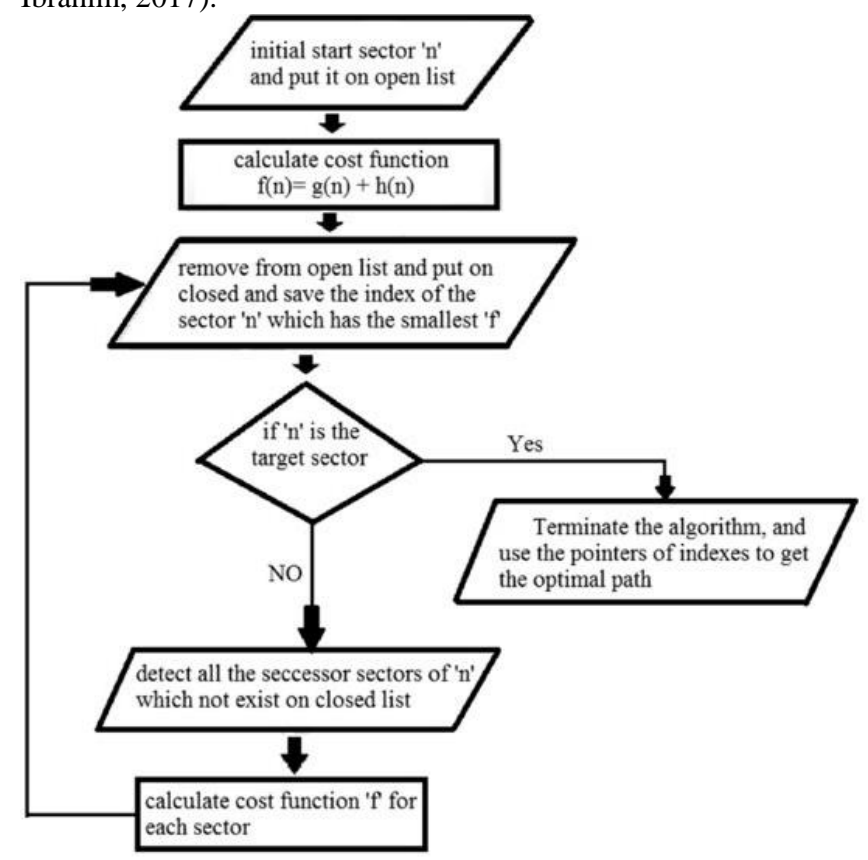

Figure 1. The flowchart of $\mathrm{A}^{*}$ algorithm

\subsection{Passenger waiting time (first passenger)}

The passenger waiting time parameter is the maximum amount of time that the first passenger waits after requesting a taxi. Certainly, this parameter determines how much the first passenger waits for other travel requests to be sent to the system and asking for a trip that is near the first passenger.

\subsection{Method of selecting the second passenger}

In this research, a spatial clustering method is used; thus, a distance analysis can be implemented on the first passenger location and other agents. Then, the passengers who are near 
each other are placed in a cluster and they can participate in a shared trip.

In this study, to elect the second passenger, when the first passenger waits, the origin of the candidates for the second traveler enters the analysis of the Euclidean distance, and the distance between them is calculated based on location of the origin of the first passenger, and the same trend for the destinations also repeated, and the distance between destinations is calculated. If these two distances between the origins and destinations were less than a certain amount, the passenger would be introduced as the second passenger. This allows two passengers to travel by using an affordable taxi. The sharing path will not be too long. The concept of spatial clustering is displayed in Figure 2.

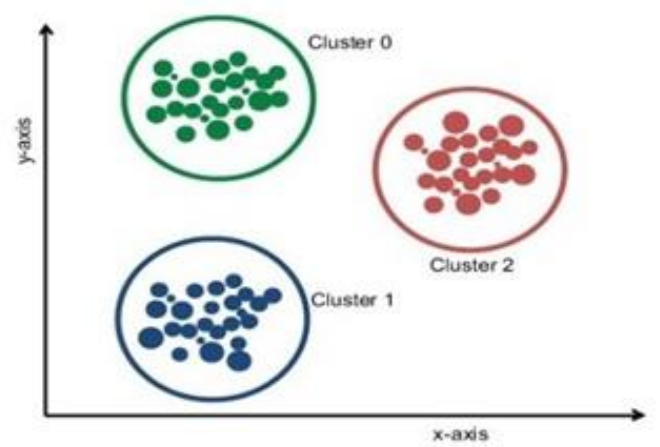

Figure 2. Measuring the closeness of agents based on Euclidean distance

\section{RESULTS}

The urban road network of Tehran city (capital of Iran in Asia) has been selected to implement the scenarios. The north-east of the city including regions $1,3,4,6,7$, and, 8 are considered as a case study which is illustrated in Figure 3.

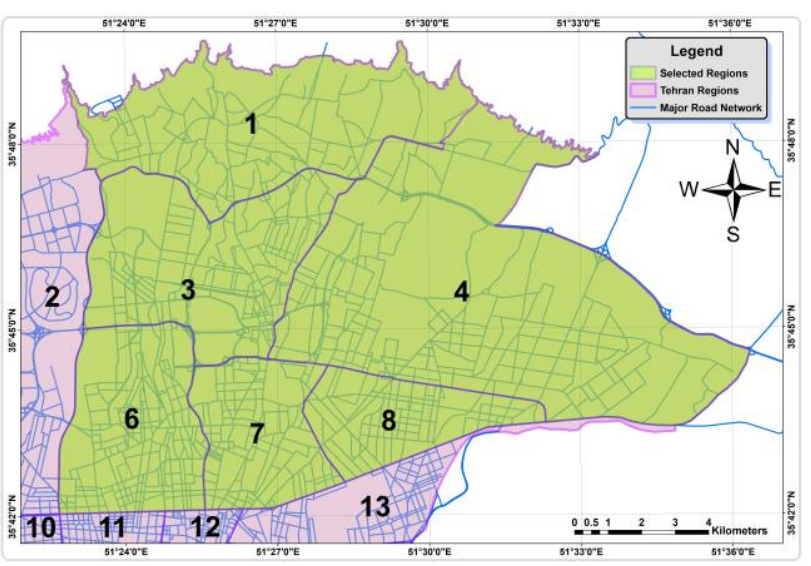

Figure 3. Tehran regions and selected case study

The network of study area is imported to the NetLogo software, and the developed model is implemented, and it is illustrated in Figure 4.

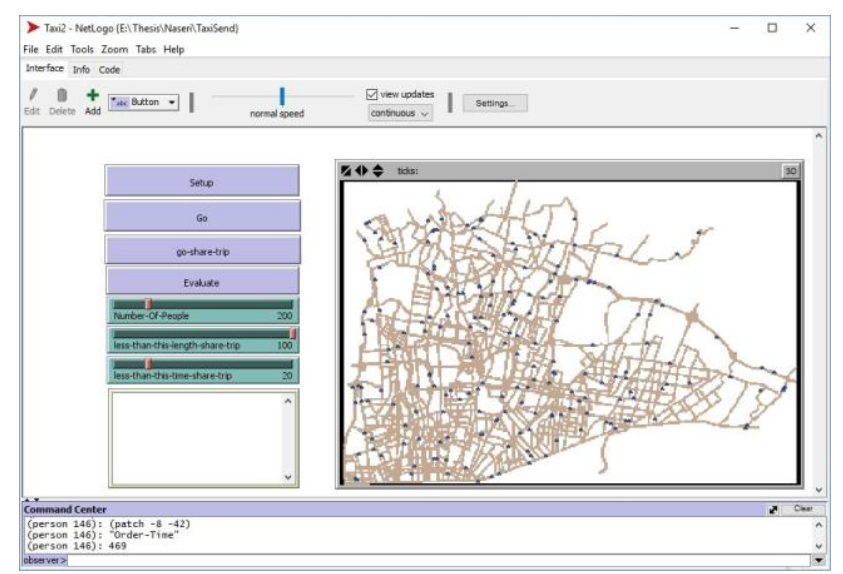

Figure 4. The NetLogo software and the customized model

In this model 3 sliders are created for model parameters, and also there are some buttons to start "individuals trip", "sharing trips", and evaluation. So, 8 scenarios are examined, and the results including "percentage of decrease in the number of trips", and "percentage of decrease in the total length of trips" are recorded. We measured changing in the number (and also total length of all trips) of vehicles which some of them carrying 2 passengers rather than when each passenger uses a vehicle. The results are displayed in Figure 5 and Figure 6.

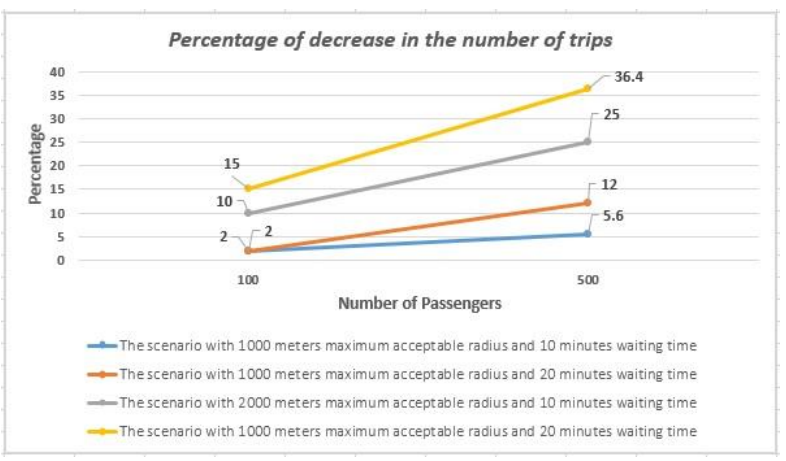

Figure 5. Percentage of decrease in the number of trips

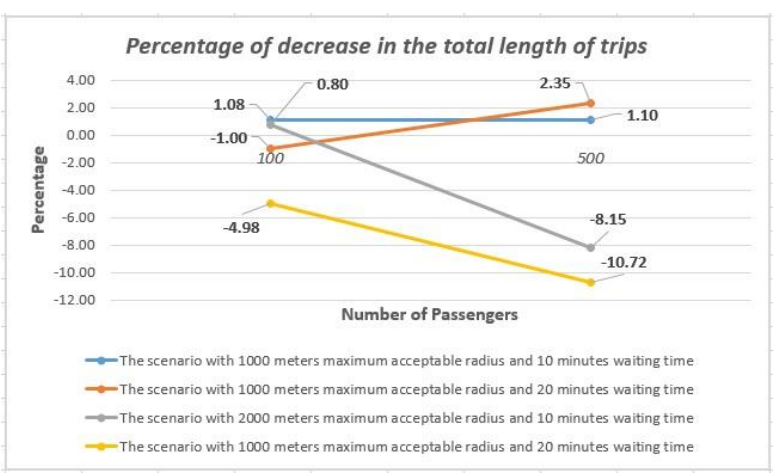

Figure 6. Percentage of decrease in the total length of trips

\section{CONCLUSION}

As it's shown in Figure 5, the number of shared travels has increased as passengers. The probability of closeness of origins and destinations are increased as the number of passengers. So, the number of shared trips are grown as the amount of the 
parameters including acceptable sharing radius and waiting time.

Besides, Figure 6 demonstrates there is no big change in total travels length in the first two scenarios, but when the acceptable radius increases, total length decreases despite increasing the shared trips. It means, not only the quantity of moving vehicles is declined and more people used shared trips, but also the algorithm found those close passengers so that the total lengths are decreased. While we expect the total travel length increases because one vehicle carries two passengers and it has to move more to take the second passenger. Furthermore, there is an abnormally, and it is related to the second scenario (the red one). Although, the waiting time increases from 10 to 20 minutes and the percentage of shared trips grows from 2 to 12 , the total travel length increases, and it means the candidates for car-sharing did not have close origins or destinations. Consequently, it proves the performance of the proposed model which finds the best matching of passengers who have common origins and destinations.

\section{REFERENCES}

Bellifemine, F.L., Caire, G. and Greenwood, D., 2007. Developing multi-agent systems with JADE (Vol. 7). John Wiley \& Sons.

Charles, K.K. and Kline, P., 2006. Relational costs and the production of social capital: evidence from carpooling. The Economic Journal, 116(511), pp.581-604.

Choset, H.M., Hutchinson, S., Lynch, K.M., Kantor, G., Burgard, W., Kavraki, L.E. and Thrun, S., 2005. Principles of robot motion: theory, algorithms, and implementation. MIT press.

Dijkstra, E.W., 1959. A note on two problems in connexion with graphs. Numerische mathematik, 1(1), pp.269-271.

Lin, Y., Li, W., Qiu, F. and Xu, H., 2012. Research on optimization of vehicle routing problem for ride-sharing taxi. Procedia-Social and Behavioral Sciences, 43, pp.494-502.

Nourinejad, M. and Roorda, M.J., 2016. Agent based model for dynamic ridesharing. Transportation Research Part C: Emerging Technologies, 64, pp.117-132.

Santos, D.O. and Xavier, E.C., 2015. Taxi and ride sharing: A dynamic dial-a-ride problem with money as an incentive. Expert Systems with Applications, 42(19), pp.6728-6737.

Simonetto, A., Monteil, J. and Gambella, C., 2019. Real-time city-scale ridesharing via linear assignment problems. Transportation Research Part C: Emerging Technologies, 101, pp.208-232.

Stiglic, M., Agatz, N., Savelsbergh, M. and Gradisar, M., 2018. Enhancing urban mobility: Integrating ride-sharing and public transit. Computers \& Operations Research, 90, pp.12-21.

Yao, J., Lin, C., Xie, X., Wang, A.J. and Hung, C.C., 2010, April. Path planning for virtual human motion using improved A* star algorithm. In 2010 Seventh international conference on information technology: new generations (pp. 1154-1158). IEEE.
Zhang, L. and Levinson, D., 2004. Agent-based approach to travel demand modeling: Exploratory analysis. Transportation Research Record, 1898(1), pp.28-36.

Zidane, I.M. and Ibrahim, K., 2017, September. Wavefront and a-star algorithms for mobile robot path planning. In International Conference on Advanced Intelligent Systems and Informatics (pp. 69-80). Springer, Cham. 